\title{
El estudio de caso en la investigación sobre la gestión de organizaciones: \\ una guía introductoria
}

Jorge Mendoza, Arturo Llaxacondor

Pontificia Universidad Católica del Perú

jmendozaw@pucp.edu.pe, arturo.llaxacondor@pucp.pe

La investigación en la gestión se enfrenta al reto de entender, construir y difundir conocimiento sobre un objeto de estudio que se presenta como algo subjetivo, difícil y complejo de abordar: las organizaciones o un conjunto de ellas. En esta situación, el estudio de caso como estrategia de investigación de fenómenos sociales se plantea como una opción relevante para el investigador, en un contexto donde el uso de estrategias intensivas sobre las extensivas mejora la calidad del entendimiento del fenómeno en relación con el entorno en donde ocurre. Bajo esta óptica, el presente documento busca brindar una visión más clara del estudio de caso en la investigación sobre la gestión de organizaciones a partir de la revisión de literatura sobre el tema y la propia experiencia de los autores, lo que responderá preguntas sobre el uso, oportunidad y factibilidad de esta estrategia.

Palabras clave: estudios de caso; metodología de investigación; gestión de organizaciones; vida organizacional; guía

The case study in organizational management research: an introductory guide

Research in organizational management faces the challenge of understanding, building and disseminating knowledge about a study object that presents itself as something subjective, difficult and complex to handle: the organizations or a group of them. In this situation, the case study as a research strategy of social phenomena is seen as a relevant option for the researcher, in a context in which the use of intensive over extensive strategies improves the quality of the understanding of this phenomenon in relation to the context in which it occurs. In this perspective, this paper seeks to provide a clearer view of the case study research on organizational management through a review of the literature on the subject and the authors' own experience on this topic, so that questions about the use, opportunity and feasibility of this strategy will be answered.

Keywords: case studies; research methodology; organizational management; organizational life; guide 


\section{Introducción}

Usualmente se considera a una organización como un objeto, el cual es manejado a través de una serie de procesos y toma de decisiones por un grupo llamado gerentes, y que es dirigido hacia un propósito previamente establecido. Pero normalmente no se toma en cuenta que dicha organización está compuesta por personas, cada una con un propósito individual y con aspiraciones y expectativas de contribuir con sus acciones tanto a su propio desarrollo como al de la organización de la que forman parte, en lo que Thorpe y Holt llaman "el reconocer la existencia empírica de la voluntad humana" (2008: 7). Tampoco se considera con frecuencia que esta organización se encuentra rodeada de un contexto con infinitos propósitos, los cuales surgen de un mundo complejo y globalizado, y que le ofrecen y exigen recursos que no son necesariamente coherentes con el propósito de la organización misma ni con el de los miembros que la conforman. Estas múltiples dimensiones y relaciones son lo que dan forma al concepto de vida organizacional, lo cual hace, por un lado, rica a la organización en experiencias y posibilidades, pero, por otro lado, hace complejo el entenderla y construir conocimiento a partir de ella.

Pensando en esa complejidad nace la idea de desarrollar este documento, como una ayuda para el investigador que busca entender, desarrollar y difundir el conocimiento sobre la gestión de organizaciones, pero en su propio contexto. En esa mirada empírica y construccionista descansa la fortaleza del estudio de caso como una estrategia de investigación. Así, este documento busca contribuir a la literatura sobre la metodología de la investigación a través de la respuesta a varias inquietudes que los investigadores de la gestión de organizaciones afrontan, como, por ejemplo, ¿cuándo es el estudio de caso una estrategia adecuada?, ¿cómo hay que enfrentarlo y prepararse para usarlo?, ¿por dónde empezar y qué hacer con lo emergente?, ¿cuál es el proceso de desarrollo del estudio de caso y cómo construirlo?, o ¿cómo asegurar la fiabilidad y robustez de la investigación con el estudio de caso? Las respuestas a estas preguntas se han elaborado a partir de la revisión de literatura sobre el tema y los aportes metodológicos que diversos autores han desarrollado y compartido, así como de las experiencias de los propios autores en los estudios de caso.

\section{La investigación en la gestión de organizaciones y su relación con el estudio de caso}

¿A qué nos referimos cuando decimos que vamos a hacer una investigación? Esta pregunta siempre viene a la mente cuando un investigador, profesor o estudiante, se enfrenta al dilema de qué investigar, por qué y cómo. La respuesta parecería fácil de desarrollar, pero en ella surgen dos dimensiones íntimamente relacionadas que es necesario tener en cuenta: por un lado, el investigador con sus preguntas y, por el otro lado, el objeto de su investigación.

En general, el motivo para investigar algo involucra el querer entender una situación o un fenómeno del cual se sabe muy poco o, si ya se conoce, se quiere expandir el conocimiento del mismo, ya sea profundizando en el tema o relacionándolo con otros campos; todo esto con el objetivo final de alcanzar un mejor entendimiento del mundo en que vivimos. Esto se da a través de la búsqueda de nuevos hechos y evidencias que surgen 
del resultado de un proceso de investigación sistemático, ordenado y empírico de la información, de la revisión crítica de las teorías existentes y/o de conducir experimentos que añadan nuevos datos a lo ya conocido (Adams y otros 2007; Bushaway 2003; Hernández, Fernández y Baptista 2010; Saunders, Lewis and Thornhill 2007).

En esta misma línea, y de acuerdo con King, Keohane y Verba, una correcta investigación científica, es decir, que use el método científico, debe presentar las siguientes cuatro características: i) debe mostrar inferencias, es decir, no solo debe ser la acumulación de datos o hechos, sino que debe buscar inferir algo sobre dicha información, lo cual no es observable directamente; ii) el procedimiento es público, lo que significa que los métodos de investigación usados deben ser explícitos, codificados y abiertos para que puedan ser evaluados, validados y replicados por otros investigadores; iii) las conclusiones son inciertas, dado que, como todo proceso de inferencia se basa en data y evidencia incierta, las conclusiones son por naturaleza incompletas, lo que permite espacios para nuevas investigaciones; y iv) el contenido es el método, dado que, como todo puede ser investigado, lo único que se mantiene constante es el conjunto de reglas de inferencia sobre las cuales se sustenta la validación del método utilizado (1994: 7-9).

Además, el investigador puede tener diferentes objetivos al diseñar una investigación, ya que esta puede ser descriptiva -busca describir la situación o fenómeno que le motiva a investigar-, explicativa -busca describir el fenómeno y explicar por qué pasó lo que pasó-, o predictiva -busca explicar el comportamiento y tratar de predecirlo para un futuro-. Esta última es la más difícil y compleja de realizar (Adams y otros 2007: 20). Thorpe y Holt, utilizando lo trabajado por el investigador social Walter Runciman, propusieron dos tipos de investigación adicionales, de reporte y evaluativa, al investigar la gestión de organizaciones (2008: 9). Los objetivos -qué se busca investigar- se pueden apreciar en la siguiente tabla.

Tabla 1: Objetivos al diseñar una investigación

\begin{tabular}{|c|c|c|c|c|c|}
\hline & De reporte & Descriptiva & Explicativa & Evaluativa & Predictiva \\
\hline $\begin{array}{l}\text { Qué se } \\
\text { pregunta }\end{array}$ & $\begin{array}{l}\text { ¿Qué existe } \\
\text { o pasó? }\end{array}$ & $\begin{array}{l}\text { ¿Cúal fue la } \\
\text { experiencia? }\end{array}$ & $\begin{array}{l}\text { ¿Por qué pasó lo que } \\
\text { pasó o por qué existe? }\end{array}$ & $\begin{array}{l}\text { ¿Fue lo } \\
\text { deseado? }\end{array}$ & $\begin{array}{l}\text { ¿Qué va a pasar? } \\
\text { ¿Lo podemos cambiar? }\end{array}$ \\
\hline
\end{tabular}

Fuente: Adaptado de Adams y otros (2007); Thorpe y Holt (2008).

Aunque el proceso de investigación presenta dichas características y alternativas, el fenómeno u objeto a ser estudiado puede involucrar infinidad de temas y disciplinas, siendo la investigación de fenómenos sociales el que nos interesa para nuestro caso y, en particular, cómo está relacionada con la investigación en la gestión de organizaciones. Con respecto a la investigación social, para Swanborn esta busca explicar fenómenos relacionados con personas, grupos, comunidades, organizaciones, naciones, etc. (2010: 1), mientras que para Ragin y Amoroso la investigación social busca construir representaciones de la vida en sociedad (2011: 8). En esa lógica, ¿qué involucra la investigación en la gestión de organizaciones? De acuerdo con Thorpe y Holt, esta busca -desde una experiencia socialentender, hacer consciente y encontrarle un significado al concepto de creación de valor, 
con el objetivo de transformar la vida en una potencialmente buena y mejor. De acuerdo con ellos, la investigación en la gestión de organizaciones:

Se preocupa de aquellos aspectos de la vida social que generalmente están relacionados con la producción y distribución de la riqueza a través de alguna forma de organización social, ya sea un emprendimiento, una empresa, una institución pública, una profesión, un oficio, etc. (2008: 1).

Desde estos puntos de vista, la investigación en la gestión de organizaciones abarca el estudio del comportamiento de personas en diferentes roles, como gerentes, clientes, empleados, propietarios, etc., además de las relaciones que se generan entre ellos; pero también implica estudiar a la organización en sí misma: la producción de sus bienes y servicios, sus operaciones y su eficiencia, cómo evolucionan, la generación o no de rentabilidad, sus políticas y su aplicación, así como la sostenibilidad de la organización en el tiempo y cómo impacta y es impactada por el entorno que la rodea. Lo anterior implica que la investigación en la gestión de organizaciones es de naturaleza multidisciplinaria, interdisciplinaria y transdisciplinaria; es decir, se nutre y necesita de conocimientos desarrollados en otras disciplinas, como la economía, la psicología, la historia y la sociología, por mencionar algunas, los cuales combina y aplica sobre la organización para desarrollar nuevos conocimientos (Cassell y Lee 2011; Greener 2008; Saunders, Lewis y Thornhill 2007).

Respecto a cómo estudiar los fenómenos sociales, Swanborn (siguiendo lo trabajado por Rom Harré) plantea que los investigadores utilizan dos tipos de acercamientos generales, los que incluyen diferentes metodologías de investigación: un acercamiento extensivo y un acercamiento intensivo (2010: 1).

Se denomina acercamiento extensivo a la búsqueda y recolección de información de las propiedades relevantes de un gran número de ocurrencias de un fenómeno. En esta forma de investigación, se obtienen conclusiones a partir de la utilización de información de una gran cantidad de situaciones, personas u organizaciones involucradas, comunidades, naciones, etc., siendo la principal estrategia de recolección de datos el uso de encuestas. Los resultados obtenidos de esta manera no tienen valor unitario, es decir, no se usan para entender el fenómeno en un individuo en particular, sino que son los agregados de todas las respuestas los que construyen las relaciones entre las variables de estudio, y permiten, de esta manera, explicar el fenómeno. En el acercamiento intensivo, el investigador se focaliza en solo una instancia específica del fenómeno a ser estudiado, o en un grupo pequeño de estas, a fin de investigarlo a profundidad. Con esta forma de acercarse al fenómeno, el investigador profundiza cada evento en su propio contexto y con mucho mayor detalle que en un acercamiento extensivo, ya que utiliza diferentes estrategias para recoger la información (entrevistas, observaciones, documentos, etc.). Tanto en el acercamiento intensivo como en el extensivo se trabaja con múltiples variables, pero solo en el primero estas se monitorean constantemente durante el tiempo para describir y explicar el comportamiento del fenómeno. Los estudios de caso son la estrategia de investigación indicada para este acercamiento (Swanborn 2010: 2). 


\title{
3. Qué es un estudio de caso, qué lo caracteriza y qué ventajas tiene
}

Varios autores han señalado que no existe consenso alrededor de una definición exacta de qué es un estudio de caso, y que bajo este nombre se hace referencia a una gran variedad de trabajos de investigación no homogéneos en metodología y propósito (Bassey 1999; Dawes 2012; Dul y Hak 2008; Remenyi 2013). Como un ejemplo de esto se ha construido la siguiente tabla, donde se puede apreciar una serie definiciones que aportan diferentes contenidos sobre lo que se considera que es un estudio de caso.

\section{Tabla 2: Objetivos al diseñar una investigación}

\begin{abstract}
El estudio de caso es una investigación empírica que investiga fenómenos contemporáneos en profundidad y en sus contextos reales, especialmente cuando las fronteras entre el fenómeno y su contexto no son claramente evidentes. Un estudio de caso se enfrenta con situaciones en donde hay más variables de interés que datos. Como resultado de esto, se basa en múltiples fuentes de evidencias cuya data usualmente converge a través de una triangulación, y se beneficia del desarrollo previo de teorías que guían el recojo y análisis de dicha información (Yin 2014: 16).
\end{abstract}

Un caso de estudio es una investigación en la cual a) un caso o un pequeño grupo de casos (estudio comparativo) son seleccionados en su contexto actual, y b) donde la información obtenida de esos casos es analizada de una manera cualitativa (Dul y Hak 2008: 4).

La esencia de un caso de estudio, la tendencia central de todos los tipos de estudio de casos, es que tratan de iluminar la decisión o un grupo de decisiones. ¿Por qué fueron tomadas? ¿Cómo fueron implementadas? ¿Cuáles fueron sus resultados? (Schramm 1971, citado en Yin 2014: 15)

Un estudio de caso es una investigación que se focaliza en describir, entender, predecir y/o controlar un individuo (por ejemplo, un proceso, un animal, una persona, una familia, una organización, un grupo, una industria, una cultura o una nación), no está limitado a un fenómeno contemporáneo, y cuyo propósito es alcanzar un profundo entendimiento de los actores, sus interacciones, sentimientos y conductas en un proceso específico durante un tiempo determinado (Woodside 2010: 1).

Una investigación que usa el estudio de caso busca responder preguntas específicas sobre individuos, grupos, instituciones, comunidades, etc. a través de un rango diferente de evidencias, las mismas que son extraídas y recogidas para buscar las mejores respuestas a dichas preguntas. Una característica fundamental es que no se empieza con nociones teóricas a priori, porque, hasta que no se involucre uno en el caso y entienda su contexto, no se pude saber qué teorías y explicaciones funcionan mejor o le dan sentido a la situación (Gillham 2000: 2).

Un caso de estudio es un término genérico de la investigación de un individuo, grupo o fenómeno. Mientras que las técnicas usadas en la investigación pueden ser varias, e incluyen acercamientos cuantitativos y cualitativos, su principal cualidad es la creencia de que un sistema humano desarrolla características de un todo o de una integridad, y que, por tanto, no es una simple colección de rasgos. Como consecuencia de esto, para poder explicar por qué las cosas ocurren como tales y para generalizar o predecir a partir de un solo ejemplo, se requiere de una profunda investigación de las interdependencias de las partes y de los patrones que de ellas emergen (citado en Bassey 1999: 26).

El estudio de caso es una investigación intensiva de una unidad singular con el propósito de entender un grupo más grande de unidades similares. Esta unidad implica un fenómeno espacialmente delimitado (una persona, una nación, un proceso social, etc.) y es observado durante un momento o en un periodo de tiempo (Gerring 2004: 342).

Fuente: Adaptado de Bassey (1999), Dul y Hak (2008), Gerring (2004), Gillham (2000), Woodside (2010) y Yin (2014). 
Tomando en cuenta estas definiciones y sus contenidos comunes, para este documento hemos definido el estudio de caso de la siguiente manera:

Una estrategia de investigación empírica, intensiva y en profundidad, que tiene como principal característica el estudio amplio de un aspecto de la realidad en su contexto real, relativamente delimitado y con patrones identificables, y que utiliza diversas fuentes de información como la revisión de documentos, entrevistas, encuestas, observación directa, etc.

Con el estudio de caso es posible realizar investigaciones para responder las preguntas presentadas en la tabla 1 que ayuden, por un lado, a la construcción de conocimiento aplicado para los profesionales en ejercicio en la gestión de organizaciones y, por otro, a la construcción de teoría para los estudios sobre dicha disciplina.

Para la construcción de dicho conocimiento, y por su carácter intensivo, el estudio de caso se enfoca en un " $n$ " pequeño, con frecuencia un solo caso, lo cual no significa necesariamente que no pueda ser grande en complejidad. Aunque la investigación se enfoca en una situación única y singular, el estudio de caso involucra un alto grado de complejidad, pues es necesario mantener una mirada holística (Gerring 2004: 342; Stake 2005: 448). En la siguiente tabla, se pueden apreciar varios ejemplos en los que se muestra el grado de complejidad que pueden alcanzar los estudios de caso dependiendo de si involucran un actor o múltiples actores; asimismo, se incluyen las distintas disciplinas en las que usualmente estos se han desarrollado.

\section{Tabla 3: Objetivos al diseñar una investigación}

\begin{tabular}{l|l|l|l} 
& \multicolumn{1}{|c|}{ Micro } & \multicolumn{1}{c}{ Meso } & Macro \\
\hline un actor & $\begin{array}{l}\text { Un paciente clínico (su trata- } \\
\text { miento), una biografía }\end{array}$ & $\begin{array}{l}\text { Una organización, un hospi- } \\
\text { tal, una dependencia pública }\end{array}$ & $\begin{array}{l}\text { Un sistema social como una co- } \\
\text { munidad, un pueblo, nación, etc. }\end{array}$ \\
\hline $\begin{array}{l}\text { Varios } \\
\text { actores }\end{array}$ & $\begin{array}{l}\text { Comportamiento de grupos } \\
\text { de personas en contextos } \\
\text { específicos }\end{array}$ & $\begin{array}{l}\text { Un grupo de empresas, una } \\
\text { red de servicios, un sector }\end{array}$ & $\begin{array}{l}\text { La carrera armamentista, el pro- } \\
\text { ceso de organizar una política } \\
\text { común europea }\end{array}$ \\
$\begin{array}{l}\text { Disciplinas } \\
\text { involucradas }\end{array}$ & $\begin{array}{l}\text { Ciencias de la Salud, Psico- } \\
\text { logía, Psicoanálisis }\end{array}$ & $\begin{array}{l}\text { Sociología, Antropología, } \\
\text { Historia, Gestión, Educación }\end{array}$ & Ciencias Políticas, Economía
\end{tabular}

Fuente: Adaptado de Swanborn (2010).

Respecto a lo que caracteriza a un estudio de caso, a diferencia de otras estrategias de investigación, Swanborn (2012: 12-13) plantea que este se centra en un fenómeno

- $\quad$ que se manifiesta en los límites de uno o pocos sistemas sociales como organizaciones, grupos, personas, etc.

- que se estudia en su contexto natural, es decir, no se simulan situaciones como sucede en los experimentos en los que se aíslan variables en ambientes controlados.

- en el que el investigador se focaliza en el proceso: la descripción y explicación del proceso que se desarrolla entre las personas participantes, sus valores, expectativas, 
opiniones, percepciones, controversias, decisiones, relaciones mutuas y comportamiento, o la descripción y explicación de procesos entre instituciones sociales como las organizaciones.

- en el que el investigador se guía inicialmente por una pregunta abierta general, explora la información y solo después formula preguntas de investigación más precisas, manteniendo una actitud abierta a aspectos no previstos que puedan surgir durante la investigación.

- que se estudia usando diversas fuentes de información, de las que las más importantes son los documentos disponibles, entrevistas con los participantes y observación (participativa).

- $\quad$ en el que (opcionalmente), al final de un proyecto de estudio de caso aplicado, el investigador invita a los involucrados a debatir sus perspectivas subjetivas para confrontar las conclusiones preliminares de la investigación a fin de tener una base más sólida para el reporte final de la investigación y clarificar posibles malentendidos.

Finalmente, Bassey describe un grupo de ventajas, inicialmente planteadas por Adelman y otros (1980), respecto al uso del estudio de caso como estrategia de investigación: i) es robusto en relación con la realidad pero difícil de organizar, a diferencia de otras estrategias que son débiles en relación con la realidad pero fáciles de organizar; ii) permite la generalización en una instancia, o de una instancia a una clase, gracias a la profundidad y la atención a los detalles y su complejidad; iii) por su enfoque en situaciones sociales, muestra las discrepancias y conflictos de los actores involucrados, y, por ende, puede ofrecer interpretaciones alternativas; iv) como producto, el estudio de caso se convierte en un material de archivo lo suficientemente rico para permitir futuras reinterpretaciones del fenómeno; v) sus resultados son interpretados y puestos en uso, lo que lo convierte en un llamado a la acción; y vi) es más abierto a la evaluación pública de la información, pero a expensas de la gran cantidad que se genera de la misma (1999: 23).

Es importante mencionar que los casos que se escriben con fines de investigación son diferentes a los casos que se escriben para la enseñanza. Estos últimos no tienen como fin responder a una pregunta de investigación, entender un fenómeno complejo o construir nuevo conocimiento. Su fin es pedagógico y, por tanto, generalmente describen un problema o una situación en la que una persona o conjunto de personas deben tomar una decisión.

\section{Cuándo usar un estudio de caso para la investigación en la gestión de organizaciones}

Antes de decidir el tipo de estrategia de investigación a usar, el investigador debe tener en cuenta dos aspectos principales: la necesidad de reconocer y formular adecuadamente el problema de investigación que se quiere resolver (lo que permitirá luego evaluar en qué medida esto se cumplió), y las condiciones en las cuales la investigación se desarrollará (acceso a la información, acceso a los métodos de recojo de datos, etc.) (Harrison 2002; Swanborn 2010).

Las preguntas planteadas en la tabla 1 son una ayuda para poder formular el problema de investigación; sin embargo, Swanborn (2010: 26) plantea que un estudio de caso 
es más factible i) si estas preguntas son abiertas, debido a la ausencia de conocimiento sobre el proceso a estudiar; ii) cuando las principales variables están relacionadas con valores, motivaciones o conductas de las personas (no agregados) en un periodo de tiempo; o iii) cuando se quiere contrastar las diferencias, percepciones e influencias entre personas (interacción social). Además, plantea que, para poder evaluar si el estudio de caso es la mejor estrategia para enfrentar dicho problema, es necesario adicionalmente tener en cuenta los siguientes criterios (2010: 33-34):

- Presencia de problemas de diseño: los estudios de caso permiten entender problemas emergentes y ofrecer soluciones específicas y prácticas, lo que es un insumo importante para investigaciones de tipo predictiva y aplicativa. El uso de varios casos simultáneos y el seguimiento de su evolución en el tiempo permiten testear las preguntas de investigación.

- Investigación-Acción (Action Research): el estudio de caso es la mejor estrategia dado que en este tipo de investigación no es suficiente el medir las opiniones e investigar las conductas de los involucrados en una situación, sino que adicionalmente se busca que estos se confronten entre ellos (a través de un moderador) y con los datos recopilados, lo cual añade más información relevante a la investigación.

- Rareza del fenómeno: el estudio de caso es la estrategia indicada cuando, debido a la novedad del fenómeno o a su escasez, no se tiene suficiente información sobre el problema a resolver.

- Factibilidad: las restricciones relacionadas con la investigación tienen gran relevancia al momento de escoger el estudio de caso como estrategia, dado que puede ser que sea la única manera de investigar un fenómeno o que el acceso a la información sea limitado. Al no tener control sobre el objeto de estudio o sobre el experimento a investigar, el estudio de caso termina siendo la mejor solución.

Finalmente, Tharenou, Donohue y Cooper sugieren que, al realizar una investigación en la gestión de organizaciones, el estudio de caso es lo más apropiado si lo que se va a investigar es algo que refleja el mejor ejemplo de un fenómeno (una buena práctica), un resultado exitoso o no de un proceso, o cómo la cultura de una organización afecta el desenvolvimiento de sus miembros en el tiempo (2007: 75). De acuerdo con ellos, un estudio de caso es lo indicado cuando se quiere investigar en una organización acerca de i) eventos inusuales, extremos o destacables, ii) situaciones poco conocidas, iii) eventos que cambian durante el tiempo, iv) prácticas comunes que son afectadas por la cultura de la institución, o v) eventos en los que se desarrollan procesos complejos.

\section{Tipos de estudios de caso}

Pueden existir diferentes tipos de estudios de caso si tomamos en cuenta los objetivos de investigación planteados en la tabla 1, dado que pueden desarrollarse para responder cada pregunta o combinaciones de las mismas; sin embargo, Stake presenta una división muy útil para entender cómo usualmente se utilizan los estudios de caso en una investigación catalogándolos en tres tipos: i) tipo intrínseco, que son los casos en los que el investiga- 
dor tiene como objetivo tener un mejor entendimiento de una situación en particular, no porque represente otros casos, ilumine un problema o tendencia, o se desee explorar o construir alguna teoría, sino porque su particularidad es de interés del investigador; ii) tipo instrumental, cuando el caso es trabajado para proveer información relevante sobre un tema y el caso en sí mismo es usado para facilitar su entendimiento, es decir, aunque la investigación sigue siendo a profundidad, el contexto y lo que se investiga responde a un interés extrínseco al caso; y iii) tipo colectivo, donde el investigador estudia un grupo de casos para investigar un fenómeno, los mismos que pueden ser similares o distintos, complementarios o redundantes, los cuales son escogidos para entender y teorizar acerca de un grupo más grande de casos (2005: 138).

Desde otra mirada, Dul y Hak (2008: 4) hacen la distinción de dos tipos de estudio de caso para la investigación: los que tienen orientación teórica y los que tienen orientación práctica, a los que Swanborn (2012: 35), por su parte, llama de "investigación básica" e "investigación aplicada" respectivamente. Esta diferencia determina principalmente la forma en que se realizará la investigación, la selección de la unidad de estudio y las implicaciones de su resultado.

Una investigación orientada a la teoría o investigación básica tiene como fin contribuir al desarrollo de la teoría, siendo su principal público la comunidad académica. Por su parte, la investigación orientada a la práctica o investigación aplicada tiene como fin contribuir al conocimiento de los profesionales que desarrollan una práctica específica, entendiéndose como práctica aquella situación de la vida real sobre la que un profesional tiene responsabilidad formal o informal y respecto a la cual actúa o debe actuar. Así, si una organización tiene una experiencia exitosa como resultado de la implementación de cierta práctica de gestión, una investigación orientada a la práctica probablemente buscará recolectar y evaluar sistemáticamente los hechos que puedan probar que el "éxito" de la organización es precisamente un resultado de la intervención. El criterio de éxito de este tipo de investigación está dado en la medida en que se pueda alcanzar una conclusión empíricamente correcta sobre el objeto de estudio. Una investigación orientada a la teoría, en cambio, no busca concluir nada respecto a esta práctica, sino más bien respecto a una afirmación o proposición teórica. El valor del hallazgo empírico de que una determinada intervención benefició a la organización estaría dado en la medida en que contribuya a esclarecer qué tan fuerte y generalizable es determinada explicación o proposición teórica (Dul y Hak 2008: 3032). Sin embargo, es importante no perder de vista que esta distinción se hace únicamente con fines metodológicos y que no debería verse la práctica como disociada de la teoría. En última instancia, la teoría tiene una utilidad práctica y sobre la base de la práctica es que se construye la teoría.

El proceso de investigación orientada a la teoría se realiza principalmente a través de dos actividades: la formulación de proposiciones y la realización de comprobaciones que las sustenten. En esta línea, se distinguen tres tipos de actividades que constituyen en conjunto el ciclo empírico: i) la exploración se refiere a la recolección y evaluación de información relevante proveniente de diversas fuentes prácticas y teóricas (insights de expertos, stakeholders, trabajos publicados, etc.) a fin de evaluar la mejor manera en que la investigación contribuya al desarrollo de la teoría. La exploración sola no es considerada investigación, pero sí un paso necesario. ii) La investigación para la construcción de teoría 
es la investigación que tiene como objetivo formular nuevas proposiciones basadas en la evidencia empírica que se obtiene de la observación de los casos del objeto de estudio. Y, por último, iii) la investigación para la comprobación de teoría, como su nombre lo indica, es la investigación que se plantea para probar la verdad o falsedad de proposiciones. Incluye a la investigación que busca probar teorías que ya han sido antes probadas (réplica) a fin de dar suficiente solidez empírica a la teoría (Dul y Hak 2008: 38).

El proceso de investigación orientada a la práctica, como se ha mencionado anteriormente, busca ampliar el conocimiento sobre la práctica que lleva a cabo una persona (un gerente, un emprendedor, un creador de políticas públicas, etc.) o grupo de personas (un equipo, una empresa familiar, etc.), generalmente al enfrentar una situación o problema que necesita ser clarificado. Para mapear y priorizar las necesidades de conocimiento se puede usar el "ciclo de intervención", que consiste en los siguientes pasos: i) reconocimiento del problema (se identifica y define el problema), ii) diagnóstico del problema (se buscan las causas por las que el problema existe), iii) diseño de una intervención (se diseñan acciones de intervención basadas en el diagnóstico que ayudarán a resolver el problema), iv) implementación (se implementa la intervención que se ha diseñado), y v) evaluación (se evalúa si los objetivos de la intervención se han alcanzado y en qué medida se ha resuelto el problema). Estas cinco fases del ciclo de intervención definen las fases por las que un problema evoluciona. Las necesidades de conocimiento, y por lo tanto de qué investigar, pueden priorizarse al ubicarse en qué parte de las fases del ciclo se encuentran quienes ejercen determinada práctica (Dul y Hak 2008: 54).

\section{Desarrollo de un estudio de caso}

Como mencionamos al inicio del presente artículo, el principal dilema del investigador es contestar el qué investigar, dado que no existe una metodología para definir el tema de investigación. Este puede surgir del interés particular del investigador, de la relevancia que el tema tiene para la discusión académica, del encargo de una institución, etc. Para ayudar en este proceso es útil la revisión general de publicaciones sobre el posible tema de investigación, la conversación con expertos y otros investigadores que tengan conocimiento del tema que se quiere investigar, etc.

Sin embargo, el inicio de toda investigación utilizando un estudio de caso parte de una realidad: que no existe un modelo de diseño de investigación a priori. Yin lo plantea directamente al indicar que "toda investigación empírica tiene un diseño de investigación implícito y no explícito" (2014: 28); en otras palabras, es el caso mismo el que termina definiendo el diseño de la investigación. Sin embargo, en el desarrollo de dicho diseño se deben tener en cuenta los siguientes componentes:

- Las preguntas de estudio: se debe clarificar cuál es la naturaleza de las preguntas de investigación, es decir, cuál es la forma de la pregunta en términos del quién, qué, por qué, dónde y cómo.

- Las proposiciones del estudio: es decir, aquellos temas que dirigen la atención hacia algo que debe ser estudiado en el caso. En los estudios de caso de exploración, en 
donde no se tienen proposiciones previas, se deben plantear un objetivo y criterios para ver si dicha exploración fue exitosa.

- La unidad de análisis: el caso a ser estudiado, el cual como regla general está relacionado con las preguntas de investigación iniciales.

- La lógica que vincula la data con las proposiciones: que son las distintas maneras mediante las cuales la información recopilada del caso se vincula con los temas estudiados: patrones emergentes, análisis de series-tiempo, síntesis de casos cruzados, etc.

- Los criterios para interpretar los resultados de la investigación: que en el caso de un estudio de caso implica analizar las teorías e hipótesis que rivalizan con las del estudio para poder rechazarlas luego con los hallazgos encontrados (Yin 2014: 29).

De igual manera, el desarrollo de un estudio de caso puede variar si el tipo de investigación está orientada hacia la investigación básica o hacia la investigación aplicada. Dul y Hak plantean las siguientes orientaciones como una ayuda para orientar las actividades de investigación:

- Una orientación hacia la investigación básica abarca actividades como i) la búsqueda de literatura científica relevante, en la que es recomendable el uso de bases de datos bibliográficas; ii) la identificación de sugerencias realizadas por otros investigadores sobre qué conviene investigar en más profundidad, usualmente encontrada en la parte de discusión de los ensayos académicos; iii) la identificación de proposiciones de interés en el marco teórico que se sustentó en una prueba inicial, y que necesitan de más réplicas a fin de incrementar su solidez y generalización; y iv) la discusión con expertos en el campo teórico a fin de comprobar que se haya consultado la literatura más relevante y si el "diagnóstico" de los vacíos teóricos encontrados es el correcto (2008: 33).

- La orientación hacia la investigación aplicada puede incluir actividades como i) la búsqueda de literatura sobre el tema de investigación en los medios generales (como periódicos y televisión) y en los medios especializados (como publicaciones en revistas de gestión, comercio, etc.); ii) la identificación de "problemas", es decir, aquellos asuntos que las personas u organizaciones describen como "aún no resueltos" o "difíciles", "explicaciones" (ideas sobre qué causa los problemas que afrontan las personas dentro de las organizaciones) y "soluciones" (qué es lo que estas personas consideran que debe hacerse para resolver los problemas); y iii) la discusión con las personas que trabajan en las organizaciones y que lidian con el tema de investigación a fin de identificar qué conocimiento requieren específicamente para actuar (2008: 33-34).

Finalmente, Eisenhardt (1989: 533) ha propuesto un mapa heurístico con etapas y pasos que ayudan a construir un diseño de investigación para el estudio de caso en el proceso de desarrollo de una teoría, y que puede ayudar en la investigación aplicada también, pero es necesario tener en cuenta que estas etapas son guías que ayudan al investigador a enfrentar el problema de investigación y no una secuencia obligada a seguir, la cual seguramente tendrá múltiples iteraciones hacia atrás y hacia adelante. Estas etapas se aprecian en el siguiente gráfico y se detallan a continuación, complementando lo trabajado por Eisenhardt con otros autores. 


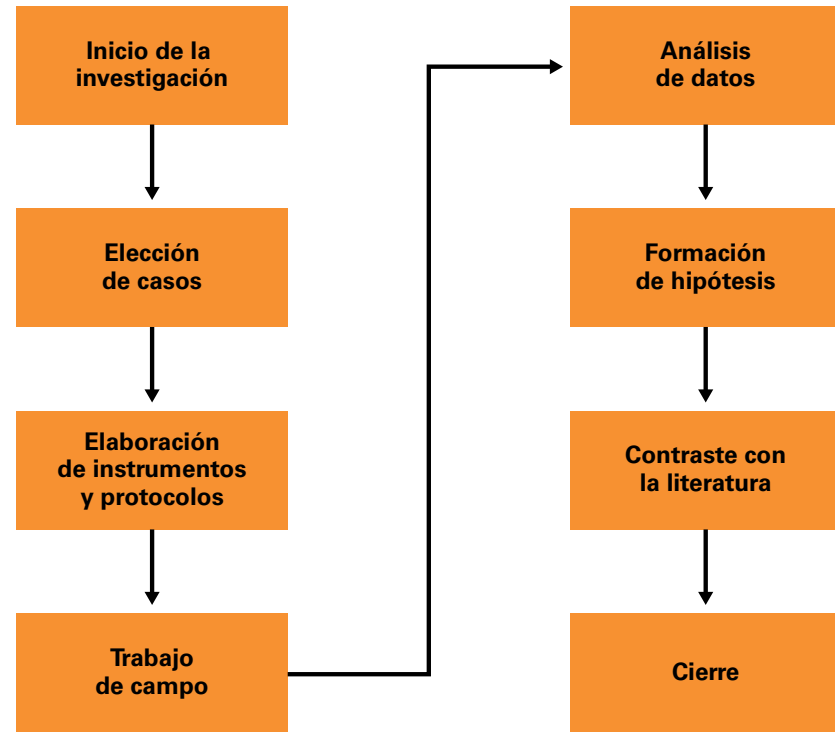

Fuente: Adaptado de Eisenhardt (1989)

a) Inicio de la investigación: al principio el investigador tiene definidas unas preguntas iniciales y trabaja posiblemente con algunos constructos a priori, no hay hipótesis y la teoría relevante es escasa, busca así mantener un grado de flexibilidad teórica para desarrollar mejor las bases para la construcción de las herramientas de medición y recolección de datos (Eisenhardt 1989: 533). Gilham plantea que en estas etapas iniciales el investigador debe haber leído alguna literatura relevante inicial, haberse informado y conocer el caso o casos que le interesa revisar en el contexto en que se sitúan, decidir los objetivos generales y empezar a darles forma a sus preguntas de investigación (2000: 16). Autores como Swanborn recomiendan empezar con preguntas amplias que deberán hacerse más específicas conforme se avance en la investigación. Sin embargo, si existiesen investigaciones previas y se quiere investigar algún aspecto con mayor detalle, es posible empezar la investigación con una pregunta más específica (2012: 29-30).

Los objetivos de la investigación se desprenden de las preguntas y es recomendable que se formulen de acuerdo a la orientación que se ha escogido para la investigación (Dul y Hak 2012: 32). Así, por ejemplo, un objetivo general de una investigación básica sería contribuir al desarrollo de la teoría respecto a un tema " $X$ " (especificando el tema de investigación), mientras que el de una investigación aplicada sería el contribuir al conocimiento del profesional " $\mathrm{P}$ " (especificando la profesión y el contexto de la vida real en que este actúa o debe actuar).

Finalmente, Hancock y Algozzine plantean que una buena manera de que el investigador se involucre con el estudio de caso es desarrollando las siguientes preguntas: i) ¿cuál es el objetivo de la investigación o del problema a resolver?, ii) ¿por qué dicho objetivo o resolver ese problema es importante?, iii) ¿qué sabemos sobre estos temas?, iv) ¿qué necesitamos saber para avanzar en el conocimiento, alcanzar el objetivo o resolver el problema?, y v) ¿cómo planeamos alcanzar el objetivo o resolver el problema? (2011: 82). 
b) Elección de casos: involucra el seleccionar un caso o casos de una manera específica y no aleatoria, buscando restringir las variaciones externas y focalizando los esfuerzos en escoger casos útiles para replicar o extender alguna teoría (Eisenhardt 1989: 533). La elección del caso define la extensión y límites de la investigación y depende en gran medida de los objetivos que esta se haya trazado. La mayoría de veces la unidad de análisis se desprende de la misma pregunta de investigación y de cómo se ha planteado inicialmente la investigación misma (Dawes 2012: 39).

La elección del caso se hace a juicio del investigador: no responde a un muestreo estadístico, sino más bien al criterio de qué casos permiten una mayor oportunidad de aprendizaje. Respecto al número de casos en una investigación con múltiples casos, este es discrecional, teniendo en cuenta que se tendrán conclusiones de mejor calidad mientras mayor sea el número de casos, por lo que se sugiere para un estudio de casos múltiples un número no menor de 4 ni mayor de 10 (Villarreal y Landeta 2010: 39-40). Es muy importante que el investigador justifique la elección de los casos a investigar, ya sea en términos de su relevancia para la investigación, qué tanto aporta al valor probatorio o no de los argumentos y conclusiones planteadas, o de su valor en términos de tipicidad o atipicidad respecto al objetivo de la investigación.

La investigación puede diseñarse de modo que se ocupen de uno o varios casos y, dentro de estos, de una o varias unidades de análisis. Yin (2014: 50) propone cuatro tipos de diseños de estudio de casos basados en una matriz con 4 variables: holístico (una sola unidad de análisis), sentido incrustado (varias unidades de análisis), un solo caso (se analiza un único caso) y múltiples casos (se analizan dos o más casos al mismo tiempo). Gráficamente la matriz se visualiza de la siguiente manera:

\section{Gráfico 2: Diseños de estudios de caso}

\section{Un solo caso}

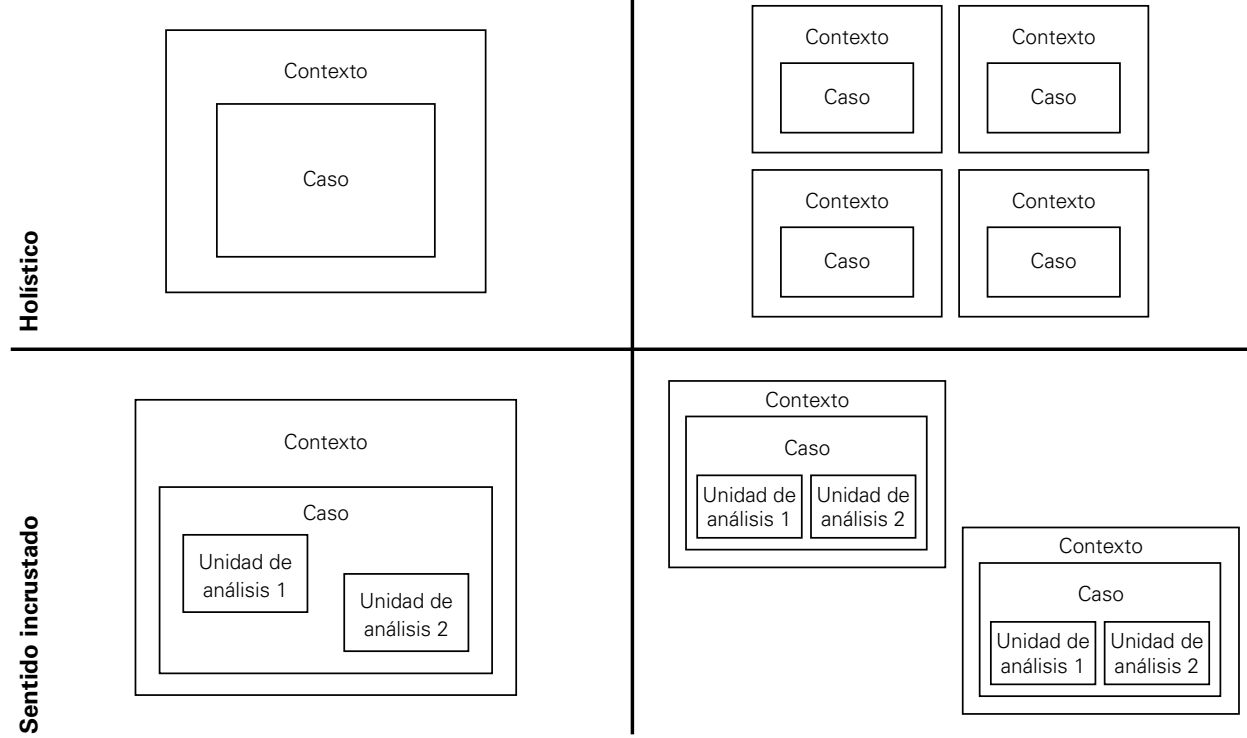


Dawes (2012: 41-43) menciona que elegir un solo caso holístico puede ser arriesgado dado que el caso podría no resultar como el investigador lo había anticipado y que la decisión de optar por este tipo de diseño tiene que ser bien sustentada a fin de no comprometer la calidad de la investigación. No obstante, una investigación de este tipo tiende a ser más profunda y reveladora, y resulta especialmente útil cuando se investiga un caso sobre un fenómeno único o de acceso restringido. Los casos de sentido incrustado, por su parte, involucran más de una unidad de análisis, aunque usualmente dentro de una misma organización (por ejemplo, podría investigarse cómo el personal de diferentes áreas percibe el servicio al consumidor). Finalmente, la opción de casos múltiples, ya sean holísticos o de sentido incrustado, permite al investigador comparar y contrastar casos o explorar un fenómeno en distintos casos y contextos.

c) Elaboración de instrumentos y protocolos: en esta etapa se busca la utilización de diferentes métodos para recolectar información que combinen data cuantitativa y cualitativa, y a través de múltiples investigadores. El objetivo es fortalecer las bases de la teoría a través de la triangulación de la evidencia y de una mirada sinérgica de la misma (Eisenhardt 1989: 533).

Asimismo, se requiere estandarizar y sistematizar los procesos de recolección de datos a fin de conferir mayor fiabilidad y validez a la investigación. Con este fin se desarrollan protocolos que deben especificar los instrumentos de recolección de datos, los procedimientos y las reglas generales que regirán el estudio de caso. Así, el protocolo se convierte en un instrumento dinámico y flexible que se puede modificar conforme el estudio va avanzando y que busca facilitar y brindar confiabilidad y validez a los datos que se recolectarán (Villarreal y Landeta 2010: 40-41). Yin menciona que el protocolo debe constar de cuatro secciones (2014: 84-85):

- Propósito general del estudio de caso. -Aquí se recoge la información general de la investigación, su contexto y perspectivas. Incluye los objetivos, la relevancia de la investigación, temas generales y el marco teórico que se empleará.

- Procedimiento de campo.- Aquí se detallan los procedimientos de campo para el recojo de información. Incluye un cronograma, los permisos para tener acceso a la organización u organizaciones involucradas en la investigación, bases de datos y otros documentos internos.

- Aspectos del estudio.- Aquí se detallan los aspectos específicos que el investigador debe tener en cuenta al momento de recoger los datos y las fuentes de información potenciales.

- Guía del informe del caso.- Aquí se determina el estilo con que se escribirá el caso, el manejo de fuentes bibliográficas y todos los elementos que facilitarán la redacción del informe final.

d) Trabajo de campo: se busca recoger y clasificar la información, tomando en cuenta el cruce de información y las notas de campo de los investigadores. Los métodos deben ser flexibles y se deben aprovechar las oportunidades que ofrece la experiencia en el campo, lo que permite al investigador tomar ventaja de los temas emergentes y las características únicas del caso (Eisenhardt 1989: 533). 
La calidad y credibilidad de la investigación recaen en gran medida en la forma en que es recolectada y procesada la información; en este sentido, este es un factor clave del éxito del estudio de caso, donde generalmente se usan datos de fuente primaria y secundaria. Los datos de fuente primaria son recolectados directamente por el investigador de fuentes originales y específicamente para el proyecto de investigación que se está conduciendo. Existen tres técnicas muy usadas en la recolección de fuentes primarias en los estudios de caso: la encuesta, la entrevista y la observación. El manejo de fuentes secundarias, por su parte, puede estar ligado al análisis de documentos proporcionados por la misma organización o por algún estudio previo realizado.

Tanto en el uso de fuentes primarias como secundarias es importante tener en cuenta dos aspectos: i) la diversidad de fuentes que caracteriza al estudio de caso (revisión de documentos, entrevistas, observación, etc.) hace más confiables los resultados de la investigación y ii) es recomendable mantener una cadena de evidencia que permita a otros investigadores reconstruir el caso siguiendo una secuencia establecida de evidencias (Villarreal y Landeta 2010: 43).

La clasificación de datos se hace con el fin de organizar la información, disponer de ella de manera más rápida, facilitar su trazabilidad y gestionarla de manera que ayude a capturar la información necesaria para responder a las preguntas de investigación. Swanborn (2012: 113-114) nos dice que, guiados por dichas preguntas, es necesario dividir la información de las fuentes de información que disponemos y archivarla en partes. Estas partes pueden identificarse como variables hasta cierto grado, y algunas pueden ser fácilmente codificadas incluso desde un inicio (precodificadas). Otras, en cambio, emergen en la investigación misma y deben ser codificadas después para que la codificación se ajuste más a la realidad. Por su parte, Dawes (2012: 93) sugiere que para esto es necesario establecer jerarquías en los códigos usando letras o números en al menos 3 niveles:

- Primer nivel.- Indica la fuente de información (por ejemplo, entrevista con el gerente general: ENT/GG).

- Segundo nivel.- Indica una sección particular de la fuente que se está usando (por ejemplo, los retos: ENT/GG - rt).

- Tercer nivel.- Indica aún un aspecto más específico sobre la sección particular de la fuente que está usando (por ejemplo, los retos con los que se está teniendo problema para lidiar: ENT/GG - rt - pb).

Los datos pueden organizarse alrededor de puntos clave, preguntas centrales o alguna categoría que ayuden al investigador a darle coherencia a la información obtenida, de tal manera que estos datos sean examinados y determinar qué tanto están relacionados con estas categorías o no, tomando en cuenta, adicionalmente, información que refute estas relaciones. Las explicaciones finales deben arrojar conclusiones basadas en una explicación que genere la mayor congruencia posible con los hechos del caso, tomando en consideración posibles explicaciones alternativas (Tharenou, Donohue y Cooper 2007: 85). Villareal adicionalmente destaca lo propuesto por Miles y Huberman (1984) al extender el análisis de los datos usando i) tablas para clasificar la información, ii) matrices de categorías para hacer un análisis cruzado de evidencias, iii) la construcción de gráficos que relacionen 
las evidencias, iv) la tabulación de frecuencias de comportamientos repetidos y sus relaciones estadísticas, y v) la clasificación de la información en orden cronológico (2010: 44).

Finalmente, en la búsqueda de patrones en el análisis de los datos encontrados, se puede seguir el "process tracing" planteado por Bennett (2010: 208), el cual tiene como objetivo el realizar inferencias causales a partir del estudio fino de evidencias que se encuentran en un menor nivel de análisis que lo planteado por la teoría relevante, para así poder establecer cuáles de estas encajan mejor en las explicaciones alternativas, como cuando un detective busca resolver un crimen uniendo diversas piezas de información para armar una explicación convincente y coherente.

f) Formación de hipótesis: para el caso de desarrollo de una teoría, en esta etapa se busca la evidencia del porqué detrás de las relaciones encontradas, confirmando o ajustando la validez teórica y construyendo la validez interna de la investigación. Las hipótesis se van formando cuando se verifica que las relaciones emergentes entre los resultados (constructos) cuadran con la evidencia encontrada en cada caso, mientras que los casos que refutan dichas relaciones brindan oportunidades para refinar y extender la teoría (Eisenhardt 1989: 542).

g) Contraste con la literatura: en el desarrollo de teoría, se busca comparar la literatura existente preguntándose qué es similar a qué, qué es lo que contradice y por qué. Examinar la literatura que confronta la teoría emergente del estudio de caso permite generar oportunidades para profundizarla y ganar nuevos insights, lo que fortalece la validez interna de la investigación y la capacidad de generalización de la misma (Eisenhardt 1989: 544-545).

Al comenzar la investigación se sugiere el uso de un marco teórico inicial que permita las primeras exploraciones y acercamientos al caso (Tharenou, Donohue y Cooper 2007: 85). Sin embargo, mientras más se profundice y progrese la investigación será necesario trabajar con otros marcos teóricos que sustenten las relaciones entre las variables emergentes, o incluso que las contradigan, para así construir mayor soporte en las premisas y los resultados de la investigación.

h) El cierre: se entiende el concepto de cierre en dos momentos: i) cuando ya no es necesario seguir añadiendo casos a la investigación y ii) cuando se detiene la iteración entre la teoría y los datos. En el primer caso, el investigador debe dejar de seguir trabajando con casos nuevos cuando se ha alcanzado un nivel de saturación teórica, es decir, cuando se tiene un incremento mínimo en el aprendizaje porque los fenómenos observados ya se han visto previamente, mientras que para el segundo caso se debe parar cuando se tiene un incremento mínimo en la mejora de la teoría (Eisenhardt 1989: 545).

\section{Redactando un estudio de caso}

No existe un formato ni estilo único para escribir un estudio de caso. Swanborn (2010: 89) plantea el uso de la narrativa analítica para redactar un estudio de caso, la misma que permitiría que se comprendan las metas y preferencias de los actores clave y las reglas que influencian sus conductas. Lo narrativo se refiere a utilizar las historias, acontecimientos y contextos en donde se reflejan las conductas de actores particulares, clarificar secuencias 
de actos, describir estructuras y explorar patrones de interacción; mientras que lo analítico hace referencia al proceso de identificar actores individuales y colectivos, sus preferencias y percepciones, su evaluación de alternativas, la información que poseen, sus expectativas y estrategias, y los obstáculos que restringen sus actos.

Autores como Remenyi (2013: 135-138) recomiendan que el estudio de caso se escriba como una historia que enganche al lector con su relato. Esta narrativa, sin embargo, debe ser "precisa y focalizada en la pregunta de investigación". Según este autor, en la redacción del estudio de caso se debe tomar en cuenta lo siguiente:

- Dar cuenta del tiempo, locación, ambiente y otros aspectos del caso incluida la pregunta de investigación, las personas involucradas y los resultados.

- Presentar a los personajes principales cuidadosamente y evitar mencionar varios personajes simultáneamente.

- Mencionar los temas principales con suficiente detalle para que el lector entienda cuál es el principal propósito de la narrativa. Una vez que los principales temas se han presentado, se debe describir la reacción de los personajes principales y los demás actores.

- Reflexionar sobre el resultado y sobre cómo debe interpretarse.

Sobre los marcos generales del estilo en que se pueden escribir los estudios de caso, Van der Blonk (Remenyi 2013: 142-145) propone al menos cuatro formas en las que la historia puede ser presentada al lector que no son mutuamente excluyentes:

- Cronológica: como su nombre lo indica, se presenta la historia de acuerdo a cómo aconteció esta en el tiempo.

- Formato de teatro: se caracteriza por tener varias secciones a modo de los actos del teatro. En cada sección debe describir la escena o escenas y los personajes principales. Los datos obtenidos generalmente son relatados por las propias voces de los informantes (que se presentan como resúmenes de las transcripciones de las entrevistas).

- Biográfica: esta forma de escritura es útil cuando la investigación se centra en cómo los individuos juegan un papel importante en los eventos que se están estudiando. Si bien es necesario el contexto en el caso, el foco está en el individuo o individuos: sus roles, motivaciones y consecuencias de sus decisiones. Son, generalmente, la forma más subjetiva de escritura.

- Recolección de voces: varios investigadores creen que las voces (palabras) de los informantes son la fuente de información más valiosa. Bajo esta premisa, varios creen que la mejor manera de escribir un caso es transcribiendo directamente lo que dicen los informantes. Una variación de esta tendencia es cuando el investigador describe en sus propias palabras lo que dicen los informantes y, luego, usa extractos de estas voces para construir sus descripciones, argumentos y afirmaciones.

Desde otra mirada, Swanborn (2010: 87) plantea que la escritura del estudio de caso puede modelar el proceso de encontrar y evaluar la evidencia siguiendo un método cuasijudicial, tomando en cuenta los siguientes momentos: 
- Los problemas iniciales y cuestiones del caso deben ser claramente definidos.

- Los antecedentes deben recogerse para proveer un contexto en donde se entiendan los problemas y las cuestiones del caso.

- Las explicaciones existentes del caso deben ser evaluadas para determinar si es que se ajustan a la evidencia, además de discernir sus carencias.

- Debería establecerse una nueva explicación que corrija los problemas identificados en las explicaciones existentes.

- Las fuentes de evidencia y la evidencia en sí misma usadas para la nueva explicación deben ser evaluadas (cross-examined).

- La coherencia interna y la lógica de la nueva explicación, incluida su compatibilidad con la evidencia, deben ser críticamente examinadas.

- Se presentan las conclusiones de la nueva explicación acerca del caso.

- Se discuten las implicancias de la nueva explicación para casos comparables.

Finalmente, sobre la estructura del documento del estudio de caso, Dawes (2012: 33-34) menciona que estos trabajos de investigación normalmente contienen tres secciones diferenciadas: el marco conceptual, el diseño de la investigación y la discusión/aporte de la investigación. Alternativamente y de manera ampliada a esta estructura básica propone la siguiente división: objetivos de la investigación, revisión de la literatura, metodología y métodos de investigación, discusión, contribución y recomendaciones para las siguientes investigaciones (2012: 117).

\section{Rigurosidad en el estudio de caso}

Es importante que el diseño del estudio de caso se haga teniendo en cuenta el criterio de triangulación (Dawes 2012; Stake 2005; Tharenou, Donohue y Cooper 2007). Por este se entiende la posibilidad de contrastar diferentes fuentes que hagan menos susceptible de error la información que se recolecta o interpreta. Existen al menos cuatro formas de triangulación, las que se explican en el siguiente cuadro:

\section{Tabla 4: Tipos de triangulación}

\begin{tabular}{c|c} 
Tipo de triangulación & \multicolumn{1}{c}{ Descripción } \\
\hline De datos & $\begin{array}{l}\text { Recolectar información con un mismo método de diferentes fuentes de infor- } \\
\text { mación y en diferentes momentos para poder captar las perspectivas de dife- } \\
\text { rentes actores y los cambios en el tiempo }\end{array}$ \\
\hline Del investigador & Usar más de un investigador en la recolección e interpretación de datos \\
\hline Teórica & Usar más de una perspectiva teórica en la interpretación de los datos \\
\hline De métodos & $\begin{array}{l}\text { Usar diferentes métodos para obtener la información, por ejemplo, realizar en- } \\
\text { trevistas y revisar documentos }\end{array}$
\end{tabular}

Fuente: Adaptado de Dawes (2012). 
Con respecto a los resultados, Dawes plantea que la validez y calidad del estudio de caso depende de si la aproximación es más positivista o más interpretativa. Para una aproximación más positivista, en donde se considera una realidad objetiva y externa, el estudio de caso debe tener las siguientes características:

- Validez del constructo: Se refiere al grado en que el estudio investiga lo que dice investigar. Se puede asegurar a través de la triangulación de fuentes de información que minimice los sesgos y del establecimiento de cadenas de evidencia que permitan al lector seguir cómo el investigador fue de la pregunta de investigación a la conclusión.

- Validez interna: Este criterio se refiere a la presencia de relaciones causales entre las variables y los resultados, y se aplica a las fases de recolección y análisis de datos. Este tipo de consideración generalmente se aplica a los casos de estudios explicativos en los que el investigador afirma que un evento " $x$ " conllevó a un evento " $y$ ". Esto puede evidenciarse detallando cómo se analizó la información.

- Confiabilidad: Se busca la ausencia de error aleatorio, de modo que, si la investigación se repitiera, los investigadores llegarían a la misma conclusión.

- Ser generalizable o validez externa: Se refiere a que las conclusiones del estudio sean aplicables a otros casos. Esta es una de las críticas más comunes al estudio de caso entendido como un método que puede contribuir al conocimiento y al desarrollo de teoría. A diferencia de los experimentos que pueden aislar variables, el estudio de caso estudia fenómenos en su contexto natural, donde las variables no pueden ser aisladas. Este problema es parcialmente resuelto si se conducen investigaciones con estudios de caso múltiples. Desde otra perspectiva, el estudio de caso puede contribuir a una generalización analítica, es decir, hacer que los resultados del caso contribuyan a generalizar una teoría previamente existente (2012: 100-104).

En una aproximación más interpretativa, en donde se considera que la realidad es múltiple, relativa y construida socialmente en lugar de ser determinada objetivamente, el autor plantea que se deben presentar los siguientes criterios:

- Credibilidad: en este criterio se busca que la investigación muestre verosimilitud, es decir, que se hayan utilizado métodos apropiados y reconocidos para su realización, y que haya evidencia de la existencia, entendimiento y uso de los protocolos de investigación.

- Transferibilidad: es la medida en que los resultados de la investigación del estudio de caso pueden ser transferidos a otros contextos. Aunque este puede no haber sido un objetivo en la investigación, en la medida que se provee de la suficiente información y descripciones adecuadamente detalladas para establecer el contexto del estudio de caso, se puede desarrollar su transferibilidad para otras investigaciones, dando mayor rigurosidad a la investigación preliminar.

- Confianza: se refiere a si el investigador tuvo que hacer cambios en la manera de recoger o de analizar la data por razones que no se definieron en el diseño de la investigación. Para demostrar la confianza se sugiere que i) se describa el diseño de la investigación y su implementación, ii) se detalle la parte operativa del recojo de la información y del trabajo de campo, y iii) se reflexione y evalúe la efectividad del proceso de investigación. 
- Confirmación: el investigador necesita convencer de que no ha sido influenciado por algún valor personal o por sus inclinaciones teóricas al momento de hacer la investigación. El uso de la triangulación, la admisión del investigador sobre sus preferencias y presunciones, y el reconocimiento de deficiencias en los métodos y sus efectos potenciales en la investigación son algunas de las maneras en las que se trabaja sobre este criterio (2012: 105-108).

\section{Reflexiones finales}

El propósito principal para la elaboración de este documento fue el de ofrecer al investigador en la gestión de organizaciones una mayor claridad acerca del estudio de caso como una estrategia de investigación, de tal manera que pueda acercarse a la realidad subjetiva de la vida organizacional con el objetivo de entender, construir y difundir un conocimiento que considere relevante, útil y necesario, tanto para la propia organización como para el cuerpo de investigadores, profesionales y académicos de la gestión.

Finalmente, si revisamos nuevamente las cuatro características planteadas por King, Keohane y Verba, acerca lo que debe presentar una investigación científica, y las comparamos con lo desarrollado en este documento acerca del estudio de caso como una estrategia de investigación, podemos concluir que el estudio de caso es una correcta investigación científica, dado que permite elaborar inferencias (además de acumular datos), sus procedimientos de investigación son públicos y pueden ser evaluados, validados y replicados por otros investigadores (en contextos similares), sus conclusiones no son completas y abren campos para nuevas investigaciones, y permite estructurar y sintetizar métodos para intervenir en diferentes campos de investigación, que incluye la gestión de organizaciones.

El documento en sí está desarrollado de una manera pro-positiva hacia las ventajas y posibilidades que el estudio de caso presenta, y, aunque esta estrategia tiene críticas válidas y presenta desventajas (la capacidad de generalización y el extenso manejo de la información por mencionar algunas), el objetivo último es el promover una continua investigación sobre fenómenos que tengan relevancia, y qué mayor relevancia que el desarrollo de estudios de caso en organizaciones peruanas, pequeñas, medianas y grandes, de las distintas regiones del país y de diversos sectores, tanto públicos como privados. El desarrollo y construcción de modelos basados en una praxis sobre la gestión de organizaciones en el Perú, el conocimiento de lo que sí funciona y lo que no funciona en nuestro contexto, es una necesidad fundamental para los gestores nacionales, y el estudio de caso es una estrategia que está a la altura del reto.

Dejamos para futuros documentos el desarrollo y profundización de esta estrategia con ejemplos aplicados a la realidad nacional, donde se podrán sistematizar diferentes acercamientos metodológicos, el uso de tecnologías para el recojo y análisis de la información, el trabajo de investigación mixto cuantitativo-cualitativo, etc.; todos estos nuevos aportes que permitirán la formación de teorías y modelos explicativos basados en organizaciones peruanas y utilizando estudios de caso. 


\section{Adams, John y otros \\ 2007 \\ Research Methods for Graduate Business and \\ Social Science Students. Los Ángeles: Response. \\ Bassey, Michael \\ Las trampas del deseo. Buenos Aires: Ariel. \\ Bennet, Andrew \\ 2010 \\ Process Tracing and Casual Inference in \\ Rethinking Social Inquiry. Nueva York: \\ Rowman \& Littlefield Publishers Inc. \\ Bushaway, Robert \\ Managing Research. Maidenhead: Open \\ University Press. \\ Cassel, Catherine y Bill Lee \\ 2011 \\ Challenges and Controversies in Manage- ment Research. Nueva York: Routledge. \\ Dawes, Jillian \\ 2012 \\ Case Study Research for Business. Los \\ Ángeles: SAGE.}

\section{Dul, Jan y Tony Hak}

Case Study Methodology in Business

Research. Londres: Routledge.

\section{Eisenhardt, Kathleen M}

"Building Theories from Case Study

Research". The Academy of Management

Review. Nueva York, volumen 14, número

4, pp. 532-550

\section{Gerring, John}

"What Is a Case Study and What Is It

Good For?" American Political Science

Review. Cambridge, volumen 98, número

2, pp. 341-354.

\section{Gillham, Bill}

2000

Case Study Research Methods. Londres:

Continuum

\section{Greener, Sue}

2008

Business Research Methods. Ventus

Publishing.

\section{Hancock, Dawson R. y}

\section{Bob Algozzine}

2011 Doing Case Study Research. Segunda Edición. Nueva York: Teachers College Press.

\section{Harrison, Alan}

"Case Study Research". Essential Skills for Management Research. Londres: SAGE. pp. 158-180.

\section{Hernández, Roberto, Carlos Fernández y María Del Pilar Baptista}

Metodología de la investigación. Quinta

Edición. México D.F.: McGraw Hill.

\section{King, Gary, Robert O. Keohane y Sidney Verba}

1994

Designing Social Inquiry. Nueva Jersey:

Princeton University Press.

\section{Ragin, Charles C. y}

Lisa M. Amoroso

2011

Constructing Social Research. Londres: SAGE.

\section{Remenyi, Dan}

2013

Case Study Research. Segunda Edición. Londres: ACPI. 


\section{bibliografía}

Saunders, Mark, Philip Lewis y Adrian Thornhill

Research Methods for Business Students.

Cuarta Edición. Harlow: Pearson.

\section{Stake, Robert}

2005

"Qualitative Case Studies". The Sage Handbook of Qualitative Research. Londres:

SAGE. pp. 443-466.

\section{Swanborn, Peter}

2010

Case Study Research. What, Why and

How? Los Ángeles: SAGE.

Taleb, Nassim

2013

Antifrágil. Las cosas que se benefician del desorden. Barcelona: Paidós.

\section{Tharenou, Phyllis, Ross \\ Donohue y Brian Cooper}

2007

Management Research Methods. Nueva

York: Cambridge University Press.

\section{Thorpe, Richard Y Robin Holt}

2008

The Sage Dictionary of Qualitative Management Research. Los Ángeles: SAGE.

\section{Villarreal, 0. y J. Landeta}

2010

“El estudio de casos como metodología de investigación científica en dirección y economía de la empresa. Una aplicación a la internacionalización." Investigaciones Europeas de Dirección y Economía de la Empresa. Volumen 16, número 3, pp. 31-52.

\section{Woodside, Arch G.}

2010 Case Study Research: Theory, Methods, Practice. Londres: Emerald.

\section{Yin, Robert K.}

2014 Case Study Research: Design and Methods. Quinta Edición. Los Ángeles: SAGE.

Fecha de recepción: 05/08/15

Fecha de aceptación: 27/10/15 